\title{
REPRESENTATION OF STATES ON EFFECT-TRIBES AND EFFECT ALGEBRAS BY INTEGRALS
}

\author{
ANATOLIJ DVUREČENSKIJ \\ 1 Mathematical Institute, Slovak Academy of Sciences \\ Štefánikova 49, SK-814 73 Bratislava, Slovakia \\ E-mail: dvurecen@mat.savba.sk,
}

\begin{abstract}
We describe $\sigma$-additive states on effect-tribes by integrals. Effecttribes are monotone $\sigma$-complete effect algebras of functions where operations are defined by points. Then we show that every state on an effect algebra is an integral through a Borel regular probability measure. Finally, we show that every $\sigma$-convex combination of extremal states on a monotone $\sigma$-complete effect algebra is a Jauch-Piron state.
\end{abstract}

\section{INTRODUCTION}

The study of the mathematical foundations of quantum mechanics was initiated by the famous article by Birkhoff and von Neumann [BiNe] and the theory was called quantum logics or in the present the theory of quantum structures. In 1993 the theory of quantum structures was enriched by effect algebras that were introduced by Foulis and Bennett FoBe. This is an algebraic structure with a partially defined primary operation, +, that model the join of two mutually excluding quantum events. It was inspired by algebraic properties of the effect operators on a Hilbert space and by POV-measures.

If an effect algebra satisfies the Riesz Decomposition Property ((RDP) for short), it is always an interval in an Abelian po-group (= partially ordered group) with interpolation and with strong unit, $[\mathrm{Rav}$. The theory of quantum structures contains Boolean algebras, orthomodular posets and lattices, orthoalgebras, etc. The monograph $[\mathrm{DvPu}]$ can serve as a comprehensive source on effect algebras.

Effect-clans form a very important family of effect algebras. They are effect algebras of $[0,1]$-valued functions with effect algebraic operations defined by points. If an effect-clan is closed with respect to pointwise limit of a sequence of nondecreasing functions, the structure is called an effect-tribe and effect-tribes are examples of monotone $\sigma$-complete effect algebras. The importance of effect-tribes is underlying by the fact that every monotone $\sigma$-complete effect algebra with (RDP) is an epimorphic image of some effect-tribe with (RDP), [BCD], an analogue of the Loomis-Sikorski Theorem.

\footnotetext{
${ }^{1}$ Keywords: Effect algebra; Riesz Decomposition Property; state; unital po-group; effect-clan; effect-tribe; simplex; Bauer simplex; integral

AMS classification: 81P15, 03G12, 03B50

The author thanks for the support by Center of Excellence SAS - Quantum Technologies -, ERDF OP R\&D Projects CE QUTE ITMS 26240120009 and meta-QUTE ITMS 26240120022, the grant VEGA No. 2/0032/09 SAV.
} 
A very important subfamily of effect algebras is a family of $M V$-algebras introduced in Cha] that model many-valued logic. They are algebraically equivalent to Phi-symmetric effect algebras that were introduced in $[\mathrm{BeFo}$. In the theory of effect algebras, MV-subalgebras of effect algebras represent so-called systems of simultaneously commensurable events of the given effect algebra, $\mathrm{DvPu}$.

In the present paper, we will study the effect-tribes in more details. We will concentrate to description of states (= analogues of probability measures) and to representation of $\sigma$-additive states by standard integrals on some appropriate $\sigma$ algebra of subsets closely connected with the tribe.

In addition, we show that every state on an effect algebra can be represented by an integral through a regular Borel probability measure on a Borel $\sigma$-algebra defined on the state space. This extends results from [Dvu4] where this was proved for interval effect algebras (= intervals in po-groups). Such a representation for states on MV-algebras was studied in [Kro, $\mathrm{Pan}$.

The paper is organized as follows. The elements of the theory of effect algebras are given in Section 2. States and a new criterion for extremal states on effect algebras are presented in Section 3. The effect-tribes and states on them are studied in Section 4, where it is shown a relation to Butnariu-Klement Theorem [BuKl] on states on tribes that are MV-algebras of functions. Section 5 deals with monotone $\sigma$ complete effect algebras and we show that every $\sigma$-convex combination of extremal states is a Jauch-Piron state. Finally, Section 6 generalizes representation of states on effect algebras by integrals through regular Borel probability measures.

\section{Effect Algebras, Effect-Clans and Effect-Tribes}

According to $\mathrm{FoBe}$, an effect algebra is a partial algebra $E=(E ;+, 0,1)$ with a partially defined operation + and two constant elements 0 and 1 such that, for all $a, b, c \in E$,

(i) $a+b$ is defined in $E$ if and only if $b+a$ is defined, and in such a case $a+b=b+a$

(ii) $a+b$ and $(a+b)+c$ are defined if and only if $b+c$ and $a+(b+c)$ are defined, and in such a case $(a+b)+c=a+(b+c)$;

(iii) for any $a \in E$, there exists a unique element $a^{\prime} \in E$ such that $a+a^{\prime}=1$;

(iv) if $a+1$ is defined in $E$, then $a=0$.

If we define $a \leq b$ if and only if there exists an element $c \in E$ such that $a+c=b$, then $\leq$ is a partial order on the set $E$, and we write $c:=b-a$. It is clear that $a^{\prime}=1-a$ for any $a \in E$.

The partial operation + denotes in fact an analogue of the join of two mutually excluding elements. For example, every Boolean algebra, orthomodular lattice, orthomodular poset, orthoalgebra is an example of effect algebras, and nowadays, effect algebras are one of the most important category of so-called quantum structures. Today, a modern approach to Hilbert space quantum mechanics, POV-measures, can be elegantly described in the frame work of effect algebras.

For a comprehensive monograph on the theory of effect algebras, see $\mathrm{DvPu}$.

There are two very important families of effect algebras, interval effect algebras, i.e., those that are an interval in a partially ordered groups (po-groups for short) and effect algebras as a system of $[0,1]$-valued functions where the effect algebraic operations are defined by points. 
Let $G$ be an Abelian po-group. An element $u \in G, u \geq 1$ is said to be a strong unit (= order unit) if for any $g \in G$, there is an integer $n \geq 1$ such that $g \leq n u$, and the couple $(G, u)$ is said to be a unital po-group. The interval $\Gamma(G, u):=[0, u]$ can be endowed with the effect algebraic operation, + , that is the restriction of the group addition + to the interval $[0, u]$; then $(\Gamma(G, u) ;+, 0, u)$ is an effect-algebra with $a^{\prime}=u-a$.

An effect algebra that is either of the form $\Gamma(G, u)$ for some element $u \geq 0$ or is isomorphic with some $\Gamma(G, u)$ is called an interval effect algebra.

For example, let $\mathcal{B}(H)$ be the system of all Hermitian operators of a Hilbert space $H$ (real, complex or quaternionic). Then $\mathcal{B}(H)$ is a po-group where the ordering of Hermitian operators is defined by $A \leq B$ iff $(A \phi, \phi) \leq(B \phi, \phi)$ for all $\phi \in H$, and the identity operator $I$ is its strong unit. Then $\mathcal{E}(H):=\Gamma(\mathcal{B}(H), I)$, the system of effect operators, is an important example of interval effect algebras used also in quantum mechanics, and

$$
\mathcal{E}(H)=\Gamma(\mathcal{B}(H), I) .
$$

An element $u \in G^{+}:=\{g \in G: g \geq 0\}$ is said to be generative if every element $g \in G^{+}$is a group sum of finitely many elements of $\Gamma(G, u)$, and $G=G^{+}-G^{+}$; then $u$ is a strong unit. If $E$ is an interval effect algebra, then there is a po-group $G$ with a generative strong unit $u$ such that $E \cong \Gamma(G, u)$ and every $H$-valued measure $p: \Gamma(G, u) \rightarrow H$ can be extended to a group-homomorphism $\phi$ from $G$ into $H$. If $H$ is a po-group, then $\phi$ is a po-group-homomorphism. Then $\phi$ is unique and $(G, u)$ is also unique up to isomorphism of unital (po-) groups, see [DvPu, Cor 1.4.21]. In such a case, the element $u$ is said to be a universal strong unit for $\Gamma(G, u)$ and the couple $(G, u)$ is said to be a unigroup. For example, the identity operator $I$ is a universal strong unit for $\Gamma(\mathcal{B}(H), I)$, [DvPu, Cor 1.4.25], similarly for $\Gamma(A, I)$, where $A$ is a von Neumann algebra on $H$.

We recall that if an effect algebra $E$ has (RDP) if $x_{1}+x_{2}=y_{1}+y_{2}$ implies there exist four elements $c_{11}, c_{12}, c_{21}, c_{22} \in E$ such that $x_{1}=c_{11}+c_{12}, x_{2}=c_{21}+c_{22}$, $y_{1}=c_{11}+c_{21}$, and $y_{2}=c_{12}+c_{22}$.

A partially ordered Abelian group $(G ;+, 0)$ (po-group in short) is said to satisfy interpolation provided given $x_{1}, x_{2}, y_{1}, y_{2}$ in $G$ such that $x_{1}, x_{2} \leq y_{1}, y_{2}$ there exists $z$ in $G$ such that $x_{1}, x_{2} \leq z \leq y_{1}, y_{2}$, and $G$ is called an interpolation group.

Ravindran Rav ( $\mathrm{DvPu}$, Thm 1.7.17]) showed that every effect algebra with (RDP) is of the form (2.1) for some interpolation unital po-group $(G, u)$. In other words, every effect algebra with (RDP) is an interval effect algebra.

Bennett and Foulis, $\mathrm{BeFo}$, introduced an important subfamily of effect algebras, Phi-symmetric effect algebras. They are equivalent to MV-algebras. We recall that an $M V$-algebra is an algebra $\left(M ; \oplus,{ }^{*}, 0\right)$ of signature $\langle 2,1,0\rangle$, where $(M ; \oplus, 0)$ is a commutative monoid with neutral element 0 , and for all $x, y \in M$

(i) $\left(x^{*}\right)^{*}=x$,

(ii) $x \oplus 1=1$, where $1=0^{*}$,

(iii) $x \oplus\left(x \oplus y^{*}\right)^{*}=y \oplus\left(y \oplus x^{*}\right)^{*}$.

If on an MV-algebra $M$ we define a partial operation, + , by $a+b$ is defined in $M$ iff $a \leq b^{*}$, and we set then $a+b:=a \oplus b$. Then $(M ;+, 0,1)$ is an interval effect algebra with (RDP), moreover, thanks to Mun, every MV-algebra is in fact an interval $\Gamma(G, u)$, where $G$ is a unital $\ell$-group (= lattice ordered group) with strong unit $u$ with $a^{*}=u-a$ and $a \oplus b:=(a+b) \wedge u, a, b \in \Gamma(G, u)$. 
The importance of MV-algebra follows from the fact if an effect algebra is a lattice, then it can be covered by blocks, maximal sets of mutually compatible elements, and each of blocks is an MV-algebra, see [Rie.

Finally we present effect algebras of $[0,1]$-valued functions with effect algebraic operations defined by points.

Let $\mathcal{E}$ be a system of $[0,1]$-valued functions on $X \neq \emptyset$ such that (i) $1 \in \mathcal{E}$, (ii) $f \in \mathcal{E}$ implies $1-f \in \mathcal{E}$, and (iii) if $f, g \in \mathcal{E}$ and $f(x) \leq 1-g(x)$ for any $x \in X$, then $f+g \in \mathcal{E}$. Then $\mathcal{E}$ is an effect algebra of functions, called an effect-clan, that is not necessarily a Boolean algebra nor an MV-algebra.

A system $\mathcal{E} \subseteq[0,1]^{X}$ is said to be a Bold algebra if (i) $1 \in \mathcal{E}$, (ii) $f \in \mathcal{E}$ implies $1-f \in \mathcal{E}$, and (iii) $f, g \in \mathcal{E}$ implies $f \oplus g \in \mathcal{E}$, where $(f \oplus g)(x):=\min \{f(x)+g(x), 1\}$, $x \in X$. Then every Bold algebra is an effect-clan with (RDP) that is an MV-algebra of functions with pointwise defined MV-operations. For example, $\chi_{A} \oplus \chi_{B}=\chi_{A \cup B}$.

An effect algebra is monotone $\sigma$-complete provided that for every ascending (descending) sequence $x_{1} \leq x_{2} \leq \cdots\left(x_{1} \geq x_{2} \geq \cdots\right)$ in $E$ which is bounded above (below) in $E$ has a supremum (infimum) in $E$.

An effect-tribe on a set $X \neq \emptyset$ is any system $\mathcal{T} \subseteq[0,1]^{X}$ such that (i) $1 \in \mathcal{T}$, (ii) if $f \in \mathcal{T}$, then $1-f \in \mathcal{T}$, (iii) if $f, g \in \mathcal{T}, f \leq 1-g$, then $f+g \in \mathcal{T}$, and (iv) for any sequences $\left\{f_{n}\right\}$ of elements of $\mathcal{T}$ such that $f_{n} \nearrow f$ (pointwisely), then $f \in \mathcal{T}$, i.e. if $f_{n}(x) \nearrow f(x)$ for every $x \in X$, then $f \in \mathcal{T}$. Every effect-tribe is a monotone $\sigma$-complete effect algebra that is also an effect-clan.

We recall that a tribe on $X \neq \emptyset$ is a collection $\mathcal{T}$ of functions from $[0,1]^{X}$ such that (i) $1 \in \mathcal{T}$, (ii) if $f \in \mathcal{T}$, then $1-f \in \mathcal{T}$, and (iii) if $\left\{f_{n}\right\}_{n}$ is a sequence from $\mathcal{T}$, then $\min \left\{\sum_{n=1}^{\infty} f_{n}, 1\right\} \in \mathcal{T}$. A tribe is always a $\sigma$-complete MV-algebra (with respect to the pointwise operations and ordering). For example, $\min \left\{\sum_{n} \chi_{A_{n}}(x), 1\right\}=$ $\chi_{\bigcup_{n} A_{n}}(x), x \in X$.

\section{States on Effect Algebra}

A state on an effect algebra $E$ is any mapping $s: E \rightarrow[0,1]$ such that (i) $s(1)=1$, and (ii) $s(a+b)=s(a)+s(b)$ whenever $a+b$ is defined in $E$.

We denote by $\mathcal{S}(E)$ the set of all states on $E$. It can happen that an effect algebra is stateless [DvPu, Ex 4.2.4]. Fortunately, every interval effect algebra has at least one state, see [Goo, Cor 4.4], in particular, this is true if $E$ has (RDP).

A state $s$ is said to be extremal if $s=\lambda s_{1}+(1-\lambda) s_{2}$ for $\lambda \in(0,1)$ implies $s=s_{1}=s_{2}$. By $\partial_{e} \mathcal{S}(E)$ we denote the set of all extremal states of $\mathcal{S}(E)$ on $E$. We say that a net of states, $\left\{s_{\alpha}\right\}$, on $E$ weakly converges to a state, $s$, on $E$ if $s_{\alpha}(a) \rightarrow s(a)$ for any $a \in E$. In this topology, $\mathcal{S}(E)$ is a compact Hausdorff topological space and every state on $E$ lies in the weak closure of the convex hull of the extremal states as it follows from the Krein-Mil'man Theorem, Goo, Thm $5.17]$.

We say that a system of states, $\mathcal{S}$, on an effect algebra $E$ is (i) order determining if $s(a) \leq s(b)$ for any $s \in \mathcal{S}$ yields $a \leq b$, and (ii) separating if, for $a, b \in E, a \neq b$, there is a state $s \in \mathcal{S}$ such that $s(a) \neq s(b)$, or, equivalently, $s(c)=s(d)$ for any $s \in \mathcal{S}$ entails $c=d$.

Let $\mathcal{S}(E) \neq \emptyset$. Given $a \in E$, we define the mapping $\hat{a}: \mathcal{S}(E) \rightarrow[0,1]$ by $\hat{a}(s):=s(a), s \in \mathcal{S}(E)$, and let $\widehat{E}:=\{\hat{a}: a \in A\}$. Then $\widehat{E}$ is an effect-clan if, e.g., $\mathcal{S}(E)$ is order determining (see [BCD1, Prop 4.1]; in such a case, $E$ and $\hat{E}$ are 
isomorphic), or $E$ is an MV-algebra; and in this case the natural mapping $\psi(a):=\hat{a}$ $(a \in E)$ is a homomorphism of $E$ onto $\widehat{E}$.

Nevertheless that $\widehat{a+b}=\hat{a}+\hat{b}, \widehat{E}$ is not necessarily an effect-clan:

Example 3.1. Let $\mathbb{Q}$ be the set of all rational numbers and let $G=\mathbb{Q} \times \mathbb{Q}$ be ordered by the strict ordering, i.e. $\left(g_{1}, g_{2}\right) \leq\left(h_{1}, h_{2}\right)$ iff $g_{1}<h_{1}$ and $g_{2}<h_{2}$ or $g_{1}=h_{1}$ and $g_{2}=h_{2}$. If we set $u=(1,1),(G, u)$ is a unital po-group with interpolation. Then for the interval effect algebra $E=\Gamma(G, u), \partial_{e} \mathcal{S}(E)=\left\{s_{0}, s_{1}\right\}$, where $s_{0}(g, h):=h$ and $s_{1}(g, h):=g, \mathcal{S}(E)$ is separating but not order determining, and $\widehat{E}$ is not an effect-clan because if $a=(0.3,0.3), b=(0.7,0.4)$, then $\hat{a} \leq 1-\hat{b}$ but $\hat{a}+\hat{b} \notin \widehat{E}$.

Let $(G, u)$ be an Abelian po-group with strong unit. By a state on $(G, u)$ we mean any mapping $s: G \rightarrow \mathbb{R}$ such that (i) $s(g+h)=s(g)+s(h)$ for all $g, h \in G$, (ii) $s\left(G^{+}\right) \subseteq \mathbb{R}^{+}$, and (iii) $s(u)=1$. In other words, a state on $(G, u)$ is any po-group homomorphism from $(G, u)$ into the po-group $(\mathbb{R}, 1)$ that preserves fixed strong units. We denote by $\mathcal{S}(G, u)$ the set of all states and by $\partial_{e} \mathcal{S}(G, u)$ the set of all extremal states, respectively, on $(G, u)$. According to Goo, Cor. 4.4], $\mathcal{S}(G, u) \neq \emptyset$ whenever $u>0$. In a similar way as for effect algebras, we define the weak convergence of states on $(G, u)$, and analogously, $\mathcal{S}(G, u)$ is a compact Hausdorff topological space and every state on $(G, u)$ is a weak limit of a net of convex combinations from $\partial_{e} \mathcal{S}(G, u)$.

If $E=\Gamma(G, u)$, where $(G, u)$ is a unigroup, then every state on $E$ can be extended to a unique state on $(G, u)$, and conversely, the restriction of any state on $(G, u)$ to $E$ gives a state on $E$. In addition, extremal states on $E$ are the restrictions of extremal states on $(G, u)$, the space $\mathcal{S}(E)$ is affinely homeomorphic with $\mathcal{S}(G, u)$, and the space $\partial_{e} \mathcal{S}(E)$ is homeomorphic with $\partial_{e} \mathcal{S}(G, u)$. We recall that a mapping from one convex set into another convex set is affine if it preserves all convex combinations.

We recall that if $E$ is an MV-algebra, then a state $s$ on $E$ is extremal if and only if $s(x \wedge y)=\min \{s(x), s(y)\}, x, y \in E$, therefore $\partial_{e} \mathcal{S}(E)$ is compact (see, e.g. [DvPu, Prop. 6.1.19], Goo, Thm 12.18]).

On the other hand, if $E$ is an effect algebra with $(\mathrm{RDP})$, then $\partial_{e} \mathcal{S}(E)$ is not necessarily compact, see for example [Goo, Exam. 6.10]. We note that due to Goo, Thm 12.14], a state $s$ on an interpolation unital po-group $(G, u)$ is extremal iff

$$
\min \{s(x), s(y)\}=\sup \left\{s(z): z \in G^{+}, z \leq x, z \leq y\right\}
$$

for all $x, y \in G^{+}$.

We recall that if $s$ is an extremal state on $E$ with (RDP), then

$$
\min \{s(x), s(y)\}=\sup \{s(z): z \in E, z \leq x, z \leq y\}
$$

for all $x, y \in E$.

We note that we do not know whether (3.2) implies (3.1). Partial positive answers are given in Proposition 3.3 and Proposition 5.3 below.

We say an effect algebra $E$ is divisible if for every positive integer $n$ and $x \in E$, there is an element $y \in E$ such that $n y=x$ and we write $y=\frac{1}{n} x$. A po-group $G$ is (i) divisible if for every positive integer $n$ and $g \in G$, there is an element $h \in G$ such that $n h=g$, (ii) unperforated if $n g \geq 0$ for some integer $n \geq 0$ implies $x \geq 0$. 
Proposition 3.2. If an effect algebra $E$ with (RDP) is divisible, then a unital pogroup $(G, u)$ with interpolation such that $E=\Gamma(G, u)$ is divisible. If an unperforated unital po-group $(G, u)$ with interpolation is divisible, then $E=\Gamma(G, u)$ is divisible.

Proof. Let $E$ be divisible. Given $g \in G^{+}$, there is an integer $k \geq 1$ such that $g \leq k u$. Then $g=g_{1}+\cdots+g_{k}$ where each $g_{i} \leq u$. For every $n \geq 1, \frac{1}{n} g_{i} \in E$, so that $g=n \frac{1}{n} g_{1}+\cdots+n \frac{1}{n} g_{k}=n\left(\frac{1}{n} g_{1}+\cdots+\frac{1}{n} g_{k}\right)$. Finally, if $g \in G$ is arbitrary, then $g=g_{1}-g_{2}$, where $g_{1}, g_{2} \geq 0$. Then $\frac{1}{n} g_{1}, \frac{1}{n} g_{2} \in G$ and hence $g=n g^{\prime}$, where $g^{\prime}=\frac{1}{n} g_{1}-\frac{1}{n} g_{2} \in G$.

Now if $G$ is divisible, then for every $x \in E=\Gamma(G, u)$ and $n \geq 1$ there is an element $y \in G$ such that $n y=x \geq 0$, hence $y \geq 0$ so that $y \in E$ and $E$ is divisible.

The following result is true also for $E=\Gamma(G, u)$, where $(G, u)$ is a unigroup.

Proposition 3.3. Let $E$ be a divisible effect algebra with (RDP). Then a state $s$ on $E$ is extremal if and only if (3.2) holds.

Proof. Let $E=\Gamma(G, u)$ for some unital po-group $(G, u)$ with interpolation. If $s$ is extremal then its unique restriction to $(G, u)$ is again extremal so that we have (3.1) and consequently (3.2).

Conversely, let (3.2) hold and assume that $s$ is not extremal. Then $s=\lambda_{1} s_{1}+$ $\lambda_{2} s_{2}$ for some distinct states $s_{1}$ and $s_{2}$ and real numbers $\lambda_{1}, \lambda_{2}>0$ such that $\lambda_{1}+\lambda_{2}=1$. We will write $s, s_{1}, s_{2}$ also for their unique extension on $(G, u)$.

Therefore, there is an element $a \in E$ such that $s_{1}(a) \neq s_{2}(a)$. Without loss of generality, we can assume $0 \leq s_{1}(a)<s_{2}(a)$. There are two positive integers $m$ and $n$ such that $s_{1}(a)<m / n<s_{2}(a)$. Put $x=n a$ and $y=m u$. Then $s_{1}(x)<s_{1}(y)$ and $s_{2}(y)<s_{2}(x)$. Hence $\frac{1}{n m} x=\frac{1}{m} a \leq a$ and $\frac{1}{n m} y \leq \frac{1}{n} u \leq u$. For any $z \in E$ with $z \leq \frac{1}{m} a$ and $z \leq \frac{1}{n} u$, we have

$$
s(z)=\lambda_{1} s_{1}(z)+\lambda_{2} s_{2}(z) \leq \lambda_{1} s_{1}\left(\frac{1}{m} a\right)+\lambda_{2} s_{2}\left(\frac{1}{n} u\right) .
$$

Hence,

$$
\min \left\{s\left(\frac{1}{m} a\right), s\left(\frac{1}{n} u\right)\right\}=\sup \left\{s(z): z \leq \frac{1}{m} a, z \leq \frac{1}{n} u\right\} \leq \lambda_{1} s_{1}\left(\frac{1}{m} a\right)+\lambda_{2} s_{2}\left(\frac{1}{n} u\right) .
$$

Since $\lambda_{1}>0$ and $s_{1}(x)<s_{1}(y)$, we have

$$
\begin{aligned}
\lambda_{1} s_{1}\left(\frac{1}{m} a\right)+\lambda_{2} s_{2}\left(\frac{1}{n} u\right) & =\lambda_{1} s_{1}\left(\frac{1}{n m} x\right)+\lambda_{2} s_{2}\left(\frac{1}{n m} y\right) \\
& =\frac{1}{n m}\left(\lambda_{1} s_{1}(x)+\lambda_{2} s_{2}(y)\right) \\
& <\frac{1}{n m}\left(\lambda_{1} s_{1}(y)+\lambda_{2} s_{2}(y)\right) \\
& =\frac{1}{n m} s(y)=s\left(\frac{1}{n} u\right) .
\end{aligned}
$$

Similarly, $\lambda_{1} s_{1}\left(\frac{1}{m} a\right)+\lambda_{2} s_{2}\left(\frac{1}{n} u\right)<s\left(\frac{1}{m} a\right)$ which gives a contradiction.

It is important to recall that according to a delicate result of Choquet Alf. Thm I.5.13], $\partial_{e} \mathcal{S}(E)$ is always a Baire space in the relative topology induced by the topology of $\mathcal{S}(E)$, i.e. the Baire Category Theorem holds for $\partial_{e} \mathcal{S}(E)$.

A $\sigma$-additive state on an effect algebra $E$ is a state $s$ such that if $a_{n} \leq a_{n+1}$ for any $n \geq 1$ and $a=\bigvee_{n} a_{n} \in E$ (and we write $a_{n} \nearrow a$ ), then $s(a)=\lim _{n} s\left(a_{n}\right)$. It is easy to verify that a state $s$ is $\sigma$-additive iff $a_{n} \searrow 0$ then $\lim _{n} s\left(a_{n}\right)=0$. 
Let $H$ be a separable Hilbert space (real, complex or quaternionic) with an inner product $(\cdot, \cdot)$, and $\mathcal{L}(H)$ be the system of all closed subspaces of $H$. Then $\mathcal{L}(H)$ is a complete orthomodular lattice $[\mathrm{Dvu}$. Given a unit vector $x \in H$, let

$$
p_{x}(M):=\left(P_{M} x, x\right), \quad M \in \mathcal{L}(H),
$$

where $P_{M}$ is the orthogonal projector of $H$ onto $M$. Then $p_{x}$ is a $\sigma$-additive state on $\mathcal{L}(H)$, called a pure state. The system of all pure states is order determining. If $T$ is a positive Hermitian operator of finite trace (i.e. $\sum_{i}\left(T x_{i}, x_{i}\right)<\infty$ for any orthonormal basis $\left\{x_{i}\right\}$ of $H$, and we define $\left.\operatorname{tr}(T):=\sum_{i}\left(T x_{i}, x_{i}\right)\right)$ such that $\operatorname{tr}(T)=1$, then

$$
s_{T}(M):=\operatorname{tr}\left(T P_{M}\right), \quad M \in \mathcal{L}(H),
$$

is a $\sigma$-additive state, and according to Gleason's theorem, Gle, Dvu, Thm 3.2.24], if $\operatorname{dim} H \geq 3$, for every $\sigma$-additive state $s$ on $\mathcal{L}(H)$, there is a unique positive Hermitian operator $T$ with $\operatorname{tr}(T)=1$ such that $s=s_{T}$.

If $s$ is a state on $\mathcal{L}(H), 3 \leq \operatorname{dim} H \leq \aleph_{0}$, then the state $s$ can be uniquely extended to a state, $\hat{s}$ on $\mathcal{B}(H)$, moreover, $\hat{s}(\alpha A)=\alpha \hat{s}(A)$ for any $\alpha \in \mathbb{C}(\alpha \in \mathbb{R})$ and $A \in \mathcal{B}(H)$.

If $\operatorname{dim} H=2$, there are two-valued states on $\mathcal{L}(H)$ and they are extremal.

We denote by $\mathcal{S}_{c}(\mathcal{L}(H))$ the system of all states that can be extended to a state on $\mathcal{B}(H)$. This is also a convex state, and if $\operatorname{dim} H \geq 3, \mathcal{S}_{c}(\mathcal{L}(H))=\mathcal{S}(\mathcal{L}(H))$.

Now if $s$ is a finitely additive state on $\mathcal{L}(H), \operatorname{dim} H \geq 3$, then due to the Aarnes theorem, [Dvu, Thm 3.2.28], $s$ can be uniquely expressed in the form

$$
s=\lambda s_{1}+(1-\lambda) s_{2},
$$

where $s_{1}$ is a $\sigma$-additive state and $s_{2}$ is a finitely additive state vanishing on each finite-dimensional subspace of $H$.

Moreover, a pure state $p_{x}$ is an extreme point of the set of $\sigma$-additive states, as well as it is an extremal state of $\mathcal{L}(H)$. Other extremal states of $\mathcal{L}(H)$ vanish on each finite-dimensional subspace of $H$. Since every state on $\mathcal{L}(H)$ can be extended into a unique state on $\mathcal{B}(H)$, see e.g. [Dvu, Thm 3.3.1], the state spaces $\mathcal{S}(\mathcal{L}(H)$ ), $\mathcal{S}(\mathcal{E}(H))$ and $\mathcal{S}(\mathcal{B}(H))$ are mutually affinely homeomorphic whenever $\operatorname{dim} H \geq 3$.

Let us set $\Omega(H):=\{x \in H:\|x\|=1\}$ and for any $A \in \mathcal{E}(H)$ we define $f_{A}(x):=(A x, x), x \in \Omega(H)$. Then $\mathcal{T}(H):=\left\{f_{A}: A \in \mathcal{E}(H)\right\}$ is an effect-tribe.

In addition, the set $\mathcal{S}=\left\{p_{x}: x \in \Omega(H)\right\}$ is an order determining system of states. The effect-algebra $\mathcal{E}(H)$ is monotone $\sigma$-complete and it is isomorphic with the effect-tribe $\widehat{\mathcal{E}(H)}$ and as well as with $\mathcal{T}(H)$.

\section{States on Effect-Tribes as Integrals}

We will characterize $\sigma$-states on effect-tribes by integrals through probability measures on a special $\sigma$-algebra of sets connected with the given tribe. We show how we can generalize the Butnariu-Klement Theorem, $\mathrm{BuKl}$, that was proved for tribes.

Let $E$ be an effect algebra. For a given element $e \in E$, we denote by $[0, e]:=$ $\{x \in E: 0 \leq x \leq e\}$. Then $[0, e]$ endowed with + restricted to $[0, e] \times[0, e]$ is an effect algebra $[0, e]=([0, e] ;+, 0, e)$. For any $x \in[0, e]$ we have $x^{\prime}{ }^{\prime}:=e-x$.

An element $e$ of an effect algebra $E$ is said to be central (or Boolean) if there exists an isomorphism

$$
f_{e}: E \rightarrow[0, e] \times\left[0, e^{\prime}\right]
$$


such that $f_{e}(e)=(e, 0)$ and if $f_{e}(x)=\left(x_{1}, x_{2}\right)$, then $x=x_{1}+x_{2}$ for any $x \in E$.

We denote by $B(E)$ the set of all central elements of $E$, and $B(E)$ is said to be the center of $E$. We recall that $0,1 \in B(E)$. According to [DvPu, Thm 1.9.14], $B(E)$ is an effect subalgebra of $E$ and it is a Boolean algebra. For any $x \in E$ and any $e \in B(E)$ we have

$$
x=(x \wedge e)+\left(x \wedge e^{\prime}\right)
$$

we define $p_{e}: E \rightarrow[0, e]$ by $p_{e}(x)=x \wedge e$.

If $E$ satisfies (RDP), then an element $e$ is central iff $e \wedge e^{\prime}=0$, Dvu1, Thm 3.2]. We note that without (RDP), the last statement is not always true: Take $X=\{1, \ldots, 6\}$ and let $\mathcal{T}$ be an effect-clan that consists of the characteristic functions of all subsets of $X$ with even numbers of elements. Then $\mathcal{T}$ is without (RDP), any element of $\mathcal{T}$ is a characteristic function but it is not always central, e.g. $\chi_{\{1,2,3,4\}} \wedge \chi_{\{1,2,3,5\}} \notin \mathcal{T}$. Moreover, $B(\mathcal{T})=\{0,1\}$.

In addition, for an effect-clan $\mathcal{T}$ with (RDP), a characteristic function $e \in \mathcal{T}$ is always a central element of $\mathcal{T}$. If $\mathcal{T}$ is even a Bold algebra, $e \in \mathcal{T}$ is central iff $e$ is a characteristic function of some subset of $X$. As we have already seen just after (4.2), without (RDP), the last statement is not always true.

In addition, if $E$ is monotone $\sigma$-complete, then $B(E)$ is a Boolean $\sigma$-algebra, [Dvu1, Thm 5.11], and for any $x \in E$ and $\left\{e_{n}\right\}$ from $B(E)$ we have $x \wedge \bigvee_{n=1}^{\infty} e_{n}=$ $\bigvee_{n=1}^{\infty}\left(x \wedge e_{n}\right)$.

Let $\mathcal{B}_{0}(\mathcal{T})$ be the system of all subsets $A$ of $X$ such that $\chi_{A}$ is a central element of the effect-clan $\mathcal{T}$, i.e.

$$
\mathcal{B}_{0}(\mathcal{T})=\left\{A \subseteq X: \chi_{A} \in B(\mathcal{T})\right\}
$$

Lemma 4.1. Let $\mathcal{T}$ be an effect-clan. If $f \in B(\mathcal{T})$ and $g \in \mathcal{T}$, then $g f \in \mathcal{T}$ and $g \wedge f=g f$, where $g f$ denotes the product of two functions $g$ and $f$.

Proof. Let $f=\chi_{A} \in B(\mathcal{T})$. We have $(g \wedge f)(x) \leq g(x)=g(x) f(x)$ if $x \in A$, and $(g \wedge f)(x) \leq f(x)=g(x) f(x)=0$ if $x \notin A$. Similarly we have also $(g \wedge(1-f))(x) \leq$ $g(x)(1-f)(x)$ for any $x \in X$.

Hence, $g(x)=(g \wedge f)(x)+(g \wedge(1-f))(x) \leq g(x) f(x)+g(x)(1-f)(x)=g(x)$, $x \in X$, which proves $g \wedge f=g f$, and $g f \in \mathcal{T}$.

The system $\mathcal{B}_{0}(\mathcal{T})$ is a $\sigma$-algebra of subsets of $X$ whenever $\mathcal{T}$ is an effect-tribe:

Proposition 4.2. Let $\mathcal{T}$ be an effect-clan. Then $\mathcal{B}_{0}(\mathcal{T})$ is an algebra of subsets, and if $E, F \in \mathcal{B}_{0}(\mathcal{T})$, then $\chi_{E} \wedge \chi_{F}=\chi_{E \cap F}$ and $\chi_{E} \vee \chi_{F}=\chi_{E \cup F}$.

If, in addition, $\mathcal{T}$ is an effect-tribe, then $\mathcal{B}(\mathcal{T})$ is a $\sigma$-algebra of subsets, and if $\left\{E_{n}\right\}$ is a sequence of elements from $\mathcal{B}_{0}(\mathcal{T})$, then $\bigwedge_{n} \chi_{E_{n}}=\chi_{\bigcap_{n} E_{n}}$ and $\bigvee_{n} \chi_{E_{n}}=$ $\chi_{\bigcup_{n} E_{n}}$.

Proof. Since $\chi_{E}$ and $\chi_{F}$ are central elements, by Lemma 4.1. $\chi_{E} \wedge \chi_{F}=\chi_{E} \chi_{F}=$ $\chi_{E \cap F}$. Passing to negations, we have the second identity.

Now let $\mathcal{T}$ be an effect-tribe and choose a sequence $\left\{E_{n}\right\}$ from $\mathcal{B}_{0}(\mathcal{T})$. According to the first part, we can assume that $E_{n} \subseteq E_{n+1}$ and let $E=\bigcup_{n} E_{n}$. Then $h=\bigvee_{n} \chi_{E_{n}} \in \mathcal{T}$ and, for any $x \in X$, we have $h(x)=\lim _{n} \chi_{E_{n}}(x)=\chi_{E}(x)$. Similarly we can prove by Lemma 4.1 that $f \chi_{E}=\lim _{n} f \chi_{E_{n}} \in \mathcal{T}$ which proves $f \chi_{E} \leq f$ and $f \chi_{e} \leq \chi_{E}$. Now if $g \leq f, \chi_{E}$ for some $g \in \mathcal{T}$, then $g \leq f \chi_{E}$ and this yields $f \wedge \chi_{E}=f \chi_{E}$. In a similar way we can prove that $f \wedge\left(1-\chi_{E}\right)=f\left(1-\chi_{E}\right) \in \mathcal{T}$ so that $f=\left(f \wedge \chi_{E}\right)+\left(f \wedge\left(1-\chi_{E}\right)\right)$ and hence $\chi_{E} \in B(\mathcal{T})$ and $E \in \mathcal{B}_{0}(\mathcal{T})$.

The second equality is now evident. 
It is worthy to recall that if $\mathcal{T}$ is an effect-clan with (RDP) then not every $f \in B(\mathcal{T})$ is a characteristic function. In fact, let $X=[0,1]$ and $\mathcal{T}=\left\{0, \operatorname{id}_{[0,1]}, 1-\right.$ $\left.\operatorname{id}_{[0,1]}, 1\right\}$. Then $\mathcal{T}$ is an MV-algebra (in fact a Boolean algebra) such that $B(\mathcal{T})=\mathcal{T}$ and $\mathcal{B}_{0}(\mathcal{T})=\{0,1\}$.

At any rate, $\mathcal{B}_{0}(\mathcal{T})$ is a Boolean sub- $\sigma$-algebra of $B(\mathcal{T})$ whenever $\mathcal{T}$ is an effecttribe.

Let $\mathcal{T}$ be an effect-clan of functions on $X \neq \emptyset$, then

$$
\mathcal{S}_{0}(\mathcal{T}):=\left\{A \subseteq X: \chi_{A} \in \mathcal{T}\right\}
$$

is a system of subsets such that (i) $\emptyset, X \in \mathcal{S}_{0}(\mathcal{T})$, (ii) if $A \in \mathcal{S}_{0}(\mathcal{T})$, then $X \backslash A \in$ $\mathcal{S}_{0}(\mathcal{T})$, and, in addition if $\mathcal{T}$ is an effect-tribe (iii) if $A_{n} \in \mathcal{S}_{0}(\mathcal{T}), A_{n} \subseteq A_{n+1}$ for any $n \geq 1$, then $A=\bigcup_{n} A_{n} \in \mathcal{S}_{0}(\mathcal{T})$. The system $\mathcal{S}_{0}(\mathcal{T})$ is not necessarily an algebra but

$$
\mathcal{B}_{0}(\mathcal{T}) \subseteq \mathcal{S}_{0}(\mathcal{T})
$$

If $\mathcal{T}$ satisfies (RDP), then $\mathcal{S}_{0}(\mathcal{T})$ is an algebra, because then any element $a=$ $\chi_{A} \in \mathcal{T}$ is central, hence, $A \in \mathcal{S}_{0}(\mathcal{T})$, and

$$
\mathcal{B}_{0}(\mathcal{T})=\mathcal{S}_{0}(\mathcal{T})
$$

Hence, $\mathcal{S}_{0}(\mathcal{T})$ is a $\sigma$-algebra of subsets whenever $\mathcal{T}$ is an effect-tribe. Indeed, because $\mathcal{S}_{0}(\mathcal{T})$ is a Boolean algebra, without loss of generality, we can assume that $\left\{A_{n}\right\}$ is a nondecreasing. Then there is $a \in \mathcal{T}$ such that $\chi_{A_{n}} \nearrow a$ by points. Hence, $a(x) \in\{0,1\}$ for any $x \in X$, so that $a=\chi_{A}$ for some $A \subseteq X$ and $\bigcup_{n} A_{n}=A \in$ $\mathcal{S}_{0}(\mathcal{T})$.

If $s$ is a finitely additive state or a $\sigma$-additive state on $\mathcal{T}$, then the mapping $\mu_{s}$ defined on $\mathcal{B}(\mathcal{T})$ by

$$
\mu_{s}(A):=s\left(\chi_{A}\right), A \in \mathcal{B}_{0}(\mathcal{T}),
$$

is a finitely additive normalized measure, or a $\sigma$-additive one on $\mathcal{B}_{0}(\mathcal{T})$.

Now we define a special type of effect-tribes. We recall that a tribe on $X \neq \emptyset$ is a collection $\mathcal{T}$ of functions from $[0,1]^{X}$ such that (i) $1 \in \mathcal{T}$, (ii) if $f \in \mathcal{T}$, then $1-f \in \mathcal{T}$, and (iii) if $\left\{f_{n}\right\}_{n}$ is a sequence from $\mathcal{T}$, then $\min \left\{\sum_{n=1}^{\infty} f_{n}, 1\right\} \in \mathcal{T}$. A tribe is always a $\sigma$-complete $\mathrm{MV}$-algebra (with respect to the pointwise operations and ordering).

Butnariu and Klement, BuKl, showed that if $\mathcal{T}$ is a tribe, then every element $f \in \mathcal{T}$ is measurable with respect to $\mathcal{B}_{0}(\mathcal{T})$ and if $s$ is a $\sigma$-additive state on $\mathcal{T}$, then

$$
s(f)=\int_{X} f \mathrm{~d} \mu_{s}, f \in \mathcal{T},
$$

where $\mu_{s}$ is as in (4.3).

It is worthy to note that in the case of the tribe $\mathcal{T}(H)$ we have $B(\mathcal{T}(H))=\{0,1\}$ and $\mathcal{B}_{0}(\mathcal{T}(H))$ is trivial, so that only constant functions from $\mathcal{T}(H)$ are $\mathcal{B}_{0}(\mathcal{T}(H))$ measurable, so that the Butnariu-Klement result is not true for all effect-tribes.

In what follows, we show that there is even an effect-tribe $\mathcal{T}$ with (RDP) that is not a lattice and not every element of $\mathcal{T}$ is measurable with respect to $\mathcal{B}_{0}(\mathcal{T})$, so that the Butnariu-Klement theorem is not necessarily valid for all effect-tribes with (RDP):

Example 4.3. There is an effect-tribe $\mathcal{T}$ with $(\mathrm{RDP})$ over a set $X \neq \emptyset$ such that $\mathcal{T}$ is not a lattice, and not every element is measurable with respect to $\mathcal{B}_{0}(\mathcal{T})$. Moreover, let $\mathcal{S}_{0}$ be the least $\sigma$-algebra of subsets of $X$ such that each $f \in \mathcal{T}$ is 
$\mathcal{S}_{0}$-measurable. There are two different probability measures, $\mu_{0}$ and $\mu_{1}$, on $\mathcal{S}_{0}$ such that formula

$$
s_{\mu}(f):=\int_{X} f \mathrm{~d} \mu, f \in \mathcal{T},
$$

defines the same $\sigma$-additive state on $\mathcal{T}$ if we set $\mu=\mu_{0}$ and $\mu=\mu_{1}$.

Proof. Let $X$ be an uncountable set with two distinct elements $a, b \in X$. Let $G$ be the set of those bounded functions $f: X \rightarrow \mathbb{R}$ satisfying $f(x)=(f(a)+f(b)) / 2$ for all but countably many $x \in X$. If 1 is a constant function 1 , then 1 is a strong unit in $G$, and according to [Goo, Ex. 16.1, Ex. 16.8], $G$ is an interpolation group, monotone $\sigma$-complete but not lattice-ordered.

Then $\mathcal{T}:=\Gamma(G, 1)$ is with (RDP), where 1 is the function equals 1 . If $\left\{f_{n}\right\}$ is a monotone sequence from $\mathcal{T}$, then $f(x)=\lim _{n} f_{n}(x), x \in X$, is a function from $\mathcal{T}$, so that $\mathcal{T}$ is an effect-tribe.

Given $x \in X, s_{x}(f):=f(x)$ for every $f \in \mathcal{T}$, is a $\sigma$-additive state on $\mathcal{T}$.

Let $\mathcal{S}$ be the $\sigma$-algebra of all subsets $A$ of $X$ such that either $A$ is countable or $X \backslash A$ is countable, and let $\mu_{0}$ be a two-valued mapping on $\mathcal{S}$ such that $\mu_{0}(A)=0$ iff $A$ is countable, otherwise $\mu_{0}(A)=1$; then $\mu_{0}$ is an extremal probability measure on $\mathcal{S}$.

Denote by $\mathcal{S}_{a, b}$ the set of all subsets $A \in \mathcal{S}$ such that if $A$ is countable then $a, b \notin A$ and if $A$ is uncountable then $a, b \in A ; \mathcal{S}_{a, b}$ is a $\sigma$-algebra.

In what follows, we show that $f \in B(\mathcal{T})$ iff $f=\chi_{A}$ where $A \in \mathcal{S}_{a, b}$, and $\mathcal{S}_{a, b}=\mathcal{B}_{0}(\mathcal{T})$.

Given $f \in \mathcal{T}$, let $A_{f}=\{x \in X: f(x)=(f(a)+f(b)) / 2\}$ and given $\alpha \in[0,1]$, let $A_{f}(\alpha)=\{x \in X: f(x) \leq \alpha\}$. Then $A_{f}=A_{1-f}$. Moreover, $a \in A_{f}$ iff $b \in A_{f}$ iff $f(a)=f(b)$.

Suppose $f \in B(\mathcal{T})$. Since $\mathcal{T}$ has $(\mathrm{RDP})$, we have that $f \wedge(1-f)=0$. The functions $f$ and $1-f$ are constant on $A_{f}$. Now let $x_{0} \in A_{f}$. If $0<f\left(x_{0}\right)<1$ define $h_{x_{0}}: X \rightarrow \mathbb{R}$ such that $h_{x_{0}}(x)=\min \{f(x), 1-f(x)\} / 2>0$ for $x \in A_{f}$ and $h_{x_{0}}(x)=0$ for $x \notin A_{f}$. Then $h_{x_{0}} \in \mathcal{T}$. Therefore, $h_{x_{0}} \leq f, 1-f$ and this contradicts the fact that $f \wedge(1-f)=0$. Consequently, either $f \backslash A_{f}=0$ or $(1-f)\left\lceil A_{f}=0\right.$. Now let $x_{0} \notin A_{f}$ then again we can show that $f\left(x_{0}\right) \in\{0,1\}$, so that $f$ is a characteristic function of a set $A \in \mathcal{B}(\mathcal{T})$ and if $a, b \in A$, then $A=A_{f}$ and if $a, b \notin A$, then $X \backslash A=A_{f}$ which yields $A \in \mathcal{S}_{a, b}$.

Conversely, if $A \in \mathcal{S}_{a, b}$ then $f=\chi_{A} \in \mathcal{T}$ and, therefore, $f \in B(\mathcal{T})$ and $A \in$ $\mathcal{B}_{0}(\mathcal{T})$.

In particular, we have proved $\mathcal{S}_{a, b}=\mathcal{B}(\mathcal{T})$.

From this we have (i) every $f \in \mathcal{T}$ is $\mathcal{S}$-measurable because $A_{f}(\alpha) \in \mathcal{S}$ for any $\alpha \in[0,1]$. (ii) Not every every $f \in \mathcal{T}$ is $\mathcal{B}_{0}(\mathcal{T})$-measurable: Indeed, choose $f \in \mathcal{T}$ such that $f(a) \neq f(b)$. Then $a, b \notin A_{f}$, but $A_{f}$ is uncountable so that $A_{f} \notin \mathcal{S}_{a, b}$. (iii) $\mathcal{S}=\mathcal{S}_{0}$.

If $\mu$ is an arbitrary probability measure on $\mathcal{S}$, then

$$
s_{\mu}(f):=\int_{X} f \mathrm{~d} \mu, f \in \mathcal{T},
$$

is a $\sigma$-additive state on $\mathcal{T}$. Of course, $s_{x}=s_{\delta_{x}}$ for any $x \in X$, where $\delta_{x}$ a Dirac measure concentrated at the point $x$, i.e. $\delta_{x}(A)=1$ iff $x \in A$ and $\delta_{x}(A)=0$ iff $x \notin A$. In particular, $s_{\mu_{0}}(f)=(f(a)+f(b)) / 2, f \in \mathcal{T}$, so that $s_{\mu_{0}}=\left(s_{a}+s_{b}\right) / 2$ 
and $s_{\mu_{0}}$ is not an extremal state on $\mathcal{T}$ however $\mu_{0}$ does on $\mathcal{S}$. In addition,

$$
s_{\mu_{0}}(f)=\int_{X} f \mathrm{~d} \mu_{0}=\int_{X} f \mathrm{~d}\left(\left(\delta_{a}+\delta_{b}\right) / 2\right), f \in \mathcal{T},
$$

so that the state $s_{\mu_{0}}$ has two different representations via (4.5): by $\mu_{0}$ and by $\mu_{1}=\left(\delta_{a}+\delta_{b}\right) / 2$.

Now we present a generalization of a result by Butnariu and Klement [BuKl] for effect-tribes with (RDP).

Theorem 4.4. Let $s$ be a $\sigma$-additive state on an effect-tribe $\mathcal{T}$ with (RDP) of functions on a set $X \neq \emptyset$ such that every $f \in \mathcal{T}$ is $\mathcal{B}_{0}(\mathcal{T})$-measurable. Then there is a unique probability measure $\mu$ on $\mathcal{B}(\mathcal{T})$ such that

$$
s(f)=\int_{X} f \mathrm{~d} \mu, f \in \mathcal{T} .
$$

Moreover, $\mu=\mu_{s}$, where $\mu_{s}$ is as in (4.3).

Proof. Set $\mu=\mu_{s}$, where $\mu_{s}$ is defined by (4.3). Since $\mathcal{T}$ satisfies (RDP), we can assume that $\mathcal{T}=\Gamma(G, 1)$ where $(G, 1)$ is an interpolation unital po-group of bounded functions on $X$. The hypothesis entails that every $f \in G$ is $\mathcal{B}_{0}(\mathcal{T})$ measurable and $s$ can be extended to a state $\hat{s}$ on $(G, u)$.

(1) Assume that $f=\chi_{A}$ is a central element of $\mathcal{T}$. Then $s(f)=s\left(\chi_{A}\right)=$ $\int_{X} \chi_{A} \mathrm{~d} \mu_{s}$.

(2) Let $f=\alpha \chi_{A}$ where $A \in \mathcal{B}_{0}(\mathcal{T})$ and $\alpha \in[0,1]$. First, let $\alpha=p / q$ for $p, q \in \mathbb{N}$. Then $q f=p \chi_{A}$ so that $\hat{s}(q f)=\hat{s}\left(p \chi_{A}\right)$ and $q s(f)=p s\left(\chi_{A}\right)=p \int_{X} \chi_{A}$ that implies $s(f)=\frac{p}{q} s\left(\chi_{A}\right)=\frac{p}{q} \int_{X} \chi_{A} \mathrm{~d} \mu_{s}$.

If $\alpha$ is irrational, there are two sequence of positive rational numbers, $\left\{p_{n} / q_{n}\right\}$ and $\left\{p_{n}^{\prime} / q_{n}^{\prime}\right\}$, such that $0 \leq p_{n} / q_{n} \nearrow \alpha \swarrow p_{n}^{\prime} / q_{n}^{\prime} \leq 1$. Then

$$
\begin{gathered}
p_{n} q_{n}^{\prime} \leq q_{n} q_{n}^{\prime} \alpha \leq q_{n} p_{n}^{\prime} \\
p_{n} q_{n}^{\prime} \chi_{A} \leq q_{n} q_{n}^{\prime} \alpha \chi_{A} \leq q_{n} p_{n}^{\prime} \chi_{A} \\
p_{n} q_{n}^{\prime} s\left(\chi_{A}\right) \leq q_{n} q_{n}^{\prime} s\left(\chi_{A}\right) \alpha \leq q_{n} p_{n}^{\prime} s\left(\chi_{A}\right) \\
\frac{p_{n}}{q_{n}} \chi_{A} \leq \alpha \chi_{A} \leq \frac{p_{n}^{\prime}}{q_{n}^{\prime}} \chi_{A},
\end{gathered}
$$

so that $s\left(\alpha \chi_{A}\right)=\alpha s\left(\chi_{A}\right)=\int_{X} \alpha \chi_{A} \mathrm{~d} \mu_{s}$.

(3) Let $f \in \mathcal{T}$. For any integer $n \geq 1$ and any $i=0,1 \ldots, 2^{n}-1$, we define $A_{n}^{i}=\left\{x \in X: \frac{i}{2^{n}}<f \leq \frac{i+1}{2^{n}}\right\} \in \mathcal{B}_{0}(\mathcal{T})$ and

$$
f_{n}:=\sum_{i=0}^{2^{n}-1} \frac{i}{2_{n}} \chi_{A_{n}^{i}}, \quad g_{n}:=\sum_{i=0}^{2^{n}-1} \frac{i+1}{2_{n}} \chi_{A_{n}^{i}} .
$$

Then $2^{n} f_{n}, 2^{n} g_{n} \in G, f_{n} \leq f \leq 2^{n} g_{n}, 2^{n} f_{n} \leq 2^{n} f \leq 2^{n} g_{n}$ and $2^{n} f_{n}, 2^{n} f, 2^{n} g_{n} \in G$. Hence,

$$
\begin{gathered}
2^{n} \sum_{i=0}^{2^{n}-1} i \chi_{A_{n}^{i}} \leq 2^{n} f \leq 2^{n} \sum_{i=0}^{2^{n}-1}(i+1) \chi_{A_{n}^{i}} \\
2^{n} \sum_{i=0}^{2^{n}-1} i s\left(\chi_{A_{n}^{i}}\right) \leq 2^{n} s(f) \leq 2^{n} \sum_{i=0}^{2^{n}-1}(i+1) s\left(\chi_{A_{n}^{i}}\right) \\
2^{n} \int_{X} f_{n} \mathrm{~d} \mu_{s} \leq s(f) \leq 2^{n} \int_{X} g_{n} \mathrm{~d} \mu_{s} \\
\int_{X} f_{n} \mathrm{~d} \mu_{s} \leq s(f) \leq \int_{X} g_{n} \mathrm{~d} \mu_{s} .
\end{gathered}
$$

Using the Lebesgue Convergence Theorem, [Hal], we have $\int_{X} \mathrm{~d} \mu_{s} \leq s(f) \leq \int_{X} f \mathrm{~d} \mu_{s}$. 
Finally, let $\mu$ be any measure on $\mathcal{B}_{0}(\mathcal{T})$ such that (4.7) holds. Then $\mu\left(\chi_{A}\right)=$ $\int_{X} \chi_{A} \mathrm{~d} \mu=\int_{X} \chi_{A} \mathrm{~d} \mu_{s}=\mu_{s}(A)$ for any $A \in \mathcal{B}_{0}(\mathcal{T})$.

Corollary 4.5. Let $\mathcal{T}$ be an effect-tribe of functions on $X \neq \emptyset$ satisfying (RDP) and let $\mathcal{T}=\Gamma(G, 1)$, where $(G, 1)$ is an interpolation unital po-group of bounded functions on a set $X \neq \emptyset$. Then every $\sigma$-additive state $s$ on $\mathcal{T}$ can be extended to a unique $\sigma$-additive state $\hat{s}$ on $(G, 1)$. Moreover,

$$
\hat{s}(f)=\int_{X} f \mathrm{~d} \mu_{s}, f \in G,
$$

where $\mu_{s}$ is defined by (4.3).

Proof. According to Theorem 4.4, we have (4.7). Since $\mathcal{T}$ generates $(G, 1)$ and it satisfies (RDP), the formula (4.7) holds for $s=\hat{s}$ and for any $f \in G$.

The $\sigma$-additivity of $\hat{s}$ on $G$ means that if $\left\{f_{n}\right\}$ is a monotone sequence of elements of $G$ such that $f_{n} \nearrow f \in G$, then $\lim _{n} \hat{s}\left(f_{n}\right)=\hat{s}(f)$. But this is guaranteed by the Lebesgue Convergence Theorem.

Remark 4.6. Theorem 4.4 and Corollary 4.5 hold also for the case that the effecttribe $\mathcal{T}$ is an interval in some unigroup $(G, u)$ of bounded functions for $\mathcal{T}$ because in this case every state on $\mathcal{T}$ can be uniquely extended to a state on $(G, u)$.

Proposition 4.7. Let $\mathcal{T}$ be an effect-tribe of functions on $X \neq \emptyset$ satisfying (RDP). Let $\mathcal{T}^{\prime}$ be the set of all functions $f \in \mathcal{T}$ such that $f$ is $\mathcal{B}_{0}(\mathcal{T})$-measurable. Then $\mathcal{T}^{\prime}$ is an effect-tribe and $\mathcal{B}_{0}(\mathcal{T})=\mathcal{B}_{0}\left(\mathcal{T}^{\prime}\right)$.

If $s$ is a $\sigma$-additive state on $\mathcal{T}$, then

$$
s(f)=\int_{X} f \mathrm{~d} \mu_{s}, f \in \mathcal{T}^{\prime},
$$

where $\mu_{s}(A):=s\left(\chi_{A}\right), A \in \mathcal{B}_{0}(\mathcal{T})$.

Proof. Due to Proposition 4.2, $\mathcal{B}_{0}(\mathcal{T})$ is a $\sigma$-algebra of subsets. Hence, $\mathcal{T}^{\prime}$ is an effect-tribe that is a subalgebra of $\mathcal{T}$. If $f=\chi_{A} \in B(\mathcal{T})$, then $\chi_{A} \in \mathcal{T}^{\prime}$. Now if $g \in \mathcal{T}^{\prime}$, due to Lemma 4.1, $g f \in \mathcal{T}$ so that $g f \in \mathcal{T}^{\prime}, g \wedge_{\mathcal{T}} f=g f=g \wedge_{\mathcal{T}} f$. Let $g=g_{1}+g_{2}$, where $g_{1}, g_{2} \in \mathcal{T}$ and $g_{1} \leq \chi_{A}, g_{2} \leq 1-\chi_{A}$, we have $g_{1}=g \chi_{A} \in \mathcal{T}^{\prime}$ and $g_{2}=g\left(1-\chi_{A}\right) \in \mathcal{T}^{\prime}$. This entails $f \in B\left(\mathcal{T}^{\prime}\right)$ and $\mathcal{B}_{0}(\mathcal{T}) \subseteq \mathcal{B}_{0}\left(\mathcal{T}^{\prime}\right)$. On the other hand, if $f=\chi_{A} \in B\left(\mathcal{T}^{\prime}\right)$, then $f \wedge(1-f)=0$ for $\wedge$ taken in $\mathcal{T}$ as well as in $\mathcal{T}^{\prime}$, so that $f \in B(\mathcal{T})$. Hence, $\mathcal{B}_{0}(\mathcal{T})=\mathcal{B}_{0}\left(\mathcal{T}^{\prime}\right)$.

Using the proof of Theorem 4.4 we have (4.9).

We note that in the case of Example 4.3. $\mathcal{T}^{\prime}$ consists from all functions $f \in \mathcal{T}$ such that $f(a)=f(b)$ and, for each $\sigma$-additive state $s$ on $\mathcal{T}$ and each $f \in \mathcal{T}^{\prime},(4.9)$ holds.

Now we apply the Butnariu-Klement Theorem for $\sigma$-additive states on a $\sigma$ complete MV-algebra.

Theorem 4.8. Let $s$ be a $\sigma$-additive state on a $\sigma$-complete $M V$-algebra $M$. Then there are a tribe $\mathcal{T}$ of functions on some $X \neq \emptyset$, a $\sigma-M V$-homomorphism $h$ from $\mathcal{T}$ onto $M$ and a unique $\sigma$-additive probability measure $\mu_{s}$ on $\mathcal{B}_{0}(\mathcal{T})=\mathcal{S}_{0}(\mathcal{T})$ such that

$$
s(a)=\int_{X} f(x) \mathrm{d} \mu_{s}(x), \quad a \in M,
$$

where $f \in \mathcal{T}$ and $h(f)=a$. 
Proof. By the Loomis-Sikorski Theorem for $\sigma$-complete MV-algebras, Dvu3, Mun, there is a tribe $\mathcal{T}$ of functions on some nonempty set $X$ and a $\sigma$-MV-homomorphism $h$ from $\mathcal{T}$ onto $M$. Define $\mathcal{B}_{0}(\mathcal{T})$, then $\mathcal{B}_{0}(\mathcal{T})=\mathcal{S}_{0}(\mathcal{T})$ and $\mathcal{B}_{0}(\mathcal{T})$ is a $\sigma$-algebra of subsets of $X$. At any rate, $h\left(\mathcal{B}_{0}(\mathcal{T})\right) \subseteq B(M)$.

Define $\mu_{s}(A)=s\left(h\left(\chi_{A}\right)\right), A \in \mathcal{B}_{0}(\mathcal{T})$, and $\hat{s}(f)=s(h(f)), f \in \mathcal{T}$. Then $\mu_{s}$ is a $\sigma$-additive probability measure on $\mathcal{B}_{0}(\mathcal{T})$ and $\hat{s}$ is a $\sigma$-additive measure on the tribe $\mathcal{T}$. By (4.4), we have

$$
\hat{s}(f)=\int_{X} f(x) \mathrm{d} \mu_{s}(x), \quad f \in \mathcal{T} .
$$

Now if $a=h(f) \in M$ for some $f \in \mathcal{T}$, then (4.11) implies (4.10).

Let $\mu$ be any $\sigma$-additive probability measure on $\mathcal{B}_{0}(\mathcal{T})$ such that (4.10) holds for $\mu$. If $A \in \mathcal{B}_{0}(\mathcal{T})$, then $\mu_{s}(A)=s\left(h\left(\chi_{A}\right)\right)=\int \chi_{A} \mathrm{~d} \mu=\mu(A)$.

Remark 4.9. (1) Theorem 4.8 holds for any tribe $\mathcal{T}$ and any $\sigma$-MV-homomorphism $h$ from $\mathcal{T}$ onto $M$.

(2) In Theorem 4.8 we can put $X=\partial_{e} \mathcal{S}(M)$ and by [Dvu3] if for any $a \in M$, we define a function $\hat{a}: \partial_{e} \mathcal{S}(M) \rightarrow[0,1]$, then $\mathcal{T}$ is a tribe generated by $\{\hat{a}: a \in M\}$ and $h(f)=a$ iff $\{x \in X: f(x)=\hat{a}(x)\}$ is a meager set. Then $h\left(\mathcal{B}_{0}(\mathcal{T})\right)=B(M)$.

\section{Monotone $\sigma$-Complete Effect Algebras}

In the present section, we characterize monotone $\sigma$-complete effect algebras analyzing their simplex structure of state spaces. We describe also Jauch-Piron states by the $\sigma$-convex hull of extremal states.

We start with some necessary definitions on convex structures. For a more detailed study we recommend [Goo, Alf, AlSc].

Let $K$ be a compact convex subset of a locally convex Hausdorff space. A mapping $f: K \rightarrow \mathbb{R}$ is said to be affine if, for all $x, y \in K$ and any $\lambda \in[0,1]$, we have $f(\lambda x+(1-\lambda) y)=\lambda f(x)+(1-\lambda) f(y)$. Let $\operatorname{Aff}(K)$ be the set of all continuous affine functions on $K$. Then $\operatorname{Aff}(K)$ is a unital po-group with the strong unit 1 which is a subgroup of the po-group $\mathrm{C}(K)$ of all continuous real-valued functions on $K$ (we recall that, for $f, g \in \mathrm{C}(K), f \leq g$ iff $f(x) \leq g(x)$ for any $x \in K$ ), hence it is an Archimedean unital po-group with the strong unit 1 . In addition, $\mathrm{C}(K)$ is an $\ell$-group (= lattice ordered group).

Let $S=\mathcal{S}(\operatorname{Aff}(K), 1)$. Then the evaluation mapping $\psi: K \rightarrow S$ defined by $\psi(x)(f)=f(x)$ for all $f \in \operatorname{Aff}(K)(x \in K)$ is an affine homeomorphism of $K$ onto $S$, see [Goo, Thm 7.1].

We recall that a convex cone in a real linear space $V$ is any subset $C$ of $V$ such that (i) $0 \in C$, (ii) if $x_{1}, x_{2} \in C$, then $\alpha_{1} x_{1}+\alpha_{2} x_{2} \in C$ for any $\alpha_{1}, \alpha_{2} \in \mathbb{R}^{+}$. A strict cone is any convex cone $C$ such that $C \cap-C=\{0\}$, where $-C=\{-x: x \in C\}$. A base for a convex cone $C$ is any convex subset $K$ of $C$ such that every non-zero element $y \in C$ may be uniquely expressed in the form $y=\alpha x$ for some $\alpha \in \mathbb{R}^{+}$and some $x \in K$.

We recall that in view of [Goo, Prop 10.2], if $K$ is a non-void convex subset of $V$ and if we set

$$
C=\left\{\alpha x: \alpha \in \mathbb{R}^{+}, x \in K\right\},
$$

then $C$ is a convex cone in $V$, and $K$ is a base for $C$ iff there is a linear functional $f$ on $V$ such that $f(K)=1$ iff $K$ is contained in a hyperplane in $V$ which misses the origin. 
Any strict cone $C$ of $V$ defines a partial order $\leq_{C}$ via $x \leq_{C} y$ iff $y-x \in C$. It is clear that $C=\left\{x \in V: 0 \leq_{C} x\right\}$. A lattice cone is any strict convex cone $C$ in $V$ such that $C$ is a lattice under $\leq_{C}$.

A simplex in a linear space $V$ is any convex subset $K$ of $V$ that is affinely isomorphic to a base for a lattice cone in some real linear space. A simplex $K$ in a locally convex Hausdorff space is said to be (i) Choquet if $K$ is compact, and (ii) Bauer if $K$ and $\partial_{e} K$ are compact.

Choquet and Bauer simplices can be characterize as follows: (i) if $E$ is with $(\mathrm{RDP})$, then $\mathcal{S}(E)$ is a Choquet simplex, Goo, Thm 10.17]. Let $K$ be a convex compact subset of a locally convex Hausdorff space, then (ii) $K$ is a Choquet simplex iff $(\operatorname{Aff}(K), 1)$ is an interpolation po-group, Goo, Thm 11.4], (iii) $\mathcal{S}(E)$ is a Bauer simplex whenever $E$ is an MV-algebra, (iv) $K$ is a Bauer simplex iff $(\operatorname{Aff}(K), 1)$ is an $\ell$-group, Goo, Thm 11.21]. (v) The state space of $\mathcal{E}(H)$ is not a simplex, BrRo, Ex 4.2.6].

We say that an effect algebra $E$ satisfies general comparability if, given $x, y \in E$, there is a central element $e \in E$ such that $p_{e}(x) \leq p_{e}(y)$ and $p_{e^{\prime}}(x) \geq p_{e^{\prime}}(y)$ where $p_{e}(x)=x \wedge e$. This means that the coordinates of the elements $x=\left(p_{e}(x), p_{e^{\prime}}(x)\right)$ and $y=\left(p_{e}(y), p_{e^{\prime}}(y)\right)$ can be compared in $[0, e]$ and $\left[0, e^{\prime}\right]$, respectively. If $E$ satisfies (RDP) and general comparability, then $E$ is a lattice, and it can be converted to an MV-algebra where original + and that derived from the MV-structure coincide.

For example, (i) every linearly ordered pseudo-effect algebra trivially satisfies general comparability; (ii) also any Cartesian product of linearly ordered pseudoeffect algebras. If $E$ satisfies general comparability, then $E$ is a lattice with (RDP) and it can be organized into an MV-algebra. In addition, every $\sigma$-complete MValgebra satisfies general comparability, [Goo, Thm 9.9]. Moreover, if $E$ satisfies general comparability, every extremal state on $B(E)$ can be uniquely extended to an extremal state on E, Goo, Thm 8.14], Dvu2, Thm 4.6].

In Example 4.3, we have a case that $\mathcal{T}$ is an effect-tribe with (RDP) such that $\mu_{0}$ is an extremal state on $B(E)$ but it has an extension to a state on $\mathcal{T}$ that is not extremal; moreover both $\mu_{0}$ and $\mu_{1}$ have the same restriction to $B(\mathcal{T})$.

In what follows, we show when the extension is unique.

We assert that if $s$ is an extremal state on $E$, then its restriction, $s_{B}=s \uparrow B(E)$, to $B(E)$ is also an extremal state on $B(E)$. Indeed, take $e \in B(E)$, we assert that $s(e) \in\{0,1\}$. If not, then $0<s(e)<1$ and $s=\lambda s_{1}+(1-\lambda) s_{2}$ where $\lambda=s(e)$, $s_{1}(x)=s(x \wedge e) / s(e)$ and $s_{2}(x)=s\left(x \wedge e^{\prime}\right) / s\left(e^{\prime}\right)$ which gives a contradiction. Therefore, $s_{B}$ is an extremal state on $B(E)$, and the mapping $\theta: \partial_{e} \mathcal{S}(E) \rightarrow$ $\partial_{e} \mathcal{S}(B(E))$ given by

$$
\theta(s)=s_{B}:=s \uparrow B(E), s \in \partial_{e} \mathcal{S}(E)
$$

is well defined and continuous.

Theorem 5.1. Let $E$ be a monotone $\sigma$-complete effect algebras with (RDP). The following statements are equivalent:

(i) Every extremal state on $B(E)$ is extendible to a unique state on $E$ and this state is extremal.

(ii) $\mathcal{S}(E)$ is a Bauer simplex.

(iii) $E$ is lattice ordered.

(iv) E satisfies general comparability. 
(v) The mapping $\theta: \partial_{e} \mathcal{S}(E) \rightarrow \partial_{e} \mathcal{S}(B(E))$ defined by (5.1) is a homeomorphism.

(vi) $E$ can be converted into an $M V$-algebra, where original + and derived one from $\oplus$ coincide.

Proof. (i) $\Rightarrow$ (ii). Let $\left\{s_{\alpha}\right\}$ be a net of extremal states on $E$ converging to a state $s \in \mathcal{S}(E)$. We assert that $s$ is extremal. It is clear that $s_{\alpha}\lceil B(E)$ converges weakly to $s_{B}:=s \uparrow B(E)$. But the space $\partial_{e} \mathcal{S}(B(E))$ is compact, so that $s_{B} \in \partial_{e} \mathcal{S}(B(E))$. Because $s_{B}$ has a unique extension, $s$, therefore, $s$ is extremal, and $\mathcal{S}(E)$ is a Bauer simplex.

According to Goo, Cor 16.28], (ii)-(iv) are equivalent.

(iv) $\Rightarrow$ (i). This follows from [Goo, Prop 8.13] or [Dvu2, Thm 4.4, Cor 4.5].

(iv) $\Rightarrow$ (v). This follows from [Goo, Thm 8.14] and

(v) $\Rightarrow$ (ii). This is evident.

(iv) $\Rightarrow$ (vi). This was already mentioned.

(vi) $\Rightarrow$ (iii). It is evident.

Now we reduce criterion (3.1) for extremality of states for monotone $\sigma$-complete effect algebras satisfying (RDP).

Proposition 5.2. Let $E=\Gamma(G, u)$ be a monotone $\sigma$-complete effect algebra satisfying (RDP). A state $s$ on $(G, u)$ is extremal if and only if, given $f, g \in G^{+}$, there is $h \in G^{+}$such that $h \leq f, g$ and $\min \{s(f), s(g)\}=s(h)$.

Proof. If the criterion is satisfied, then $s$ is extremal. Converse, let $s$ be an extremal state on $(G, u)$. Denote $s_{0}=\sup \left\{s(h): h \in G^{+}, \quad h \leq f, g\right\}=\min \{s(h), s(g)\}$. There exists a sequence of elements $\left\{h_{n}\right\}$ in $(G, u)$ such that $h_{n} \leq f, g$ and $\lim _{n} s\left(h_{n}\right)=$ $s_{0}$. Due the the interpolation property holding in the po-group $G$, there exists a monotone sequence $\left\{h_{n}^{\prime}\right\}$ such that, for any $n \geq 1, h_{1}, \ldots, h_{n} \leq h_{n}^{\prime} \leq f, g$. Let $h_{0}=\bigvee_{n} h_{n}^{\prime} \in G$, then $h_{n} \leq h_{0} \leq f, g$ so that $s\left(h_{n}\right) \leq s\left(h_{0}\right) \leq s_{0}$ and $s\left(h_{0}\right)=s_{0}$.

Proposition 5.2 can be generalized as follows.

We recall that a poset $E$ satisfies the countable interpolation property provided that for any two sequences of elements of $E,\left\{a_{i}\right\}$ and $\left\{b_{j}\right\}$, such that $a_{i} \leq b_{j}$ for all $i, j$, there is an element $c \in E$ such that $a_{i} \leq c \leq b_{j}$ for all $i, j$. Due to Goo, Prop 16.3], an effect algebra $E=\Gamma(G, u)$ with (RDP) has countable interpolation iff the po-group $G$ has countable interpolation, and due to Goo, Thm 16.10], if an effect algebra $E$ with (RDP) is monotone $\sigma$-complete, then $E$ satisfies countable interpolation.

On the other hand, there is even an MV-algebra satisfying countable interpolation that is not monotone $\sigma$-complete as we can deduce from [Goo, p. 280].

Proposition 5.3. Let $E=\Gamma(G, u)$ be an effect algebra satisfying (RDP) and countable interpolation. A state $s$ on $(G, u)$ is extremal if and only if, given $f, g \in$ $G^{+}$, there is $h \in G^{+}$such that $h \leq f, g$ and

$$
s(h)=\min \{s(f), s(g)\} .
$$

If, in addition, $E$ is divisible, then a state $s$ on $E$ is extremal if and only if (5.2) holds for all $f, g \in E$. 
Proof. One implication is evident.

Now let $s$ be an extremal state on $(G, u)$. Denote $s_{0}=\sup \left\{s(h): h \in G^{+}, \quad h \leq\right.$ $f, g\}=\min \{s(h), s(g)\}$. There exists a sequence of elements $\left\{h_{n}\right\}$ in $(G, u)$ such that $h_{n} \leq f, g$ and $\lim _{n} s\left(h_{n}\right)=s_{0}$. Due the countable interpolation property holding in the po-group $G$, there exists an element $h_{0} \in G$ such that $h_{n} \leq h_{0} \leq f, g$, for each $n$, so that $s\left(h_{n}\right) \leq s\left(h_{0}\right) \leq s_{0}$ and $s\left(h_{0}\right)=s_{0}$.

The last statement follows from Proposition 3.3 .

The latter two propositions can be used to characterize Jauch-Piron states. We say that a state $s$ on an effect algebra $E$ is Jauch-Piron if, for all $a, b \in E$ with $s(a)=s(b)=1$, there is an element $c \in E$ such that $c \leq a, c \leq b$ and $s(c)=1$. For example, if $E$ is an MV-algebra, then every state $s$ is Jauch-Piron in view of the property $s(a \vee b)+s(a \wedge b)=s(a)+s(b)$ holding in each MV-algebra. Every state in Example 3.1 is also Jauch-Piron.

We recall that if $\left\{s_{n}\right\}$ is a finite or a countable set of states, then $s=\sum_{n} \lambda_{n} s_{n}$, where $\lambda_{n} \geq 0$ and $\sum_{n} \lambda_{n}=1$, is a state that is called a $\sigma$-convex combination of $\left\{s_{n}\right\}$.

Proposition 5.4. Let $E$ be an effect algebra satisfying (RDP) and countable interpolation. Then every $\sigma$-convex combination of extremal states is Jauch-Piron.

Proof. Suppose that $s$ is an extremal state on $E$ and let $s(a)=s(b)=1$. Due to Proposition 5.3, there is an element $c \in E$ such that $c \leq a, b$ and $s(c)=$ $\min \{s(a), s(b)\}=1$.

Now let $s=\sum_{n} \lambda_{n} s_{n}$ where each $s_{n} \in \partial_{e} \mathcal{S}(E)$ and $0<\lambda_{n}<1, \sum_{n} \lambda_{n}=1$. Let $s(a)=s(b)=1$. Then $s_{n}(a)=s_{n}(b)=1$ for each $n$. Hence, there is $c_{n} \leq a, b$ such that $s_{n}\left(c_{n}\right)=1$. Due to the countable interpolation property, there is an element $c \in E$ such that $c_{n} \leq c \leq a, b$ for every $n$. Then $s_{n}\left(c_{n}\right) \leq s_{n}(c)=1$ and $s(c)=1$.

Now we present some examples to show how the extremality criterion (5.2) works on effect-clans $\mathcal{T} \subseteq[0,1]^{X}$ with (RDP) for states of the form

$$
s_{x}(f)=f(x), f \in \mathcal{T},
$$

where $x \in X$. We note that each $s_{x}$ is in fact a $\sigma$-additive state on the effect-tribe $\mathcal{T}$ and the system $\left\{s_{x}: x \in X\right\}$ is an order determining system of states.

Example 5.5. (1) Let $X=[0,1]$ be the real unit interval and $\left.E=\Gamma\left(\mathrm{C}(X), 1_{X}\right)\right)$. Since $X$ is a Hausdorff compact topological space, due to the Riesz Representation Theorem, a state $s$ is extremal iff $s=s_{x}$ for some $x \in X$, moreover, if $s_{x}(f)=$ $\int_{X} f \mathrm{~d} \mu$ where $\mu$ is the Borel probability on the Borel $\sigma$-algebra $\mathcal{B}_{0}(X)$, then $\mu=\delta_{x}$. The same is true if $X$ is any compact Hausdorff topological space and $\mu$ is a regular Borel probability measure $\mathcal{B}_{0}(X)$.

(2) Let $X=[0,1]$ be the real interval. Then $X$ is a convex compact Hausdorff topological space; set $E=\Gamma\left(\operatorname{Aff}(X), 1_{X}\right)$. Because $X$ is a simplex (for the definition of a simplex see Goo or the next chapter), $E$ is an effect-clan with (RDP), Goo, Thm 11.4]. Every element $f \in \operatorname{Aff}(X)$ is of the form $f(x)=\alpha x+\beta$ where $0 \leq \beta \leq 1$ and either $0 \leq \alpha \leq 1$ or $-1 \leq \alpha<0$. $E$ is an effect-tribe: Indeed, let $f_{n}(x)=$ $\alpha_{n} x+\beta_{n} \leq f_{n+1}(x)=\alpha_{n+1} x+\beta_{n+1}$ be bounded in $E$. Then $\beta_{n} \leq \beta_{n+1}$ so that there exists $\beta_{0}=\lim _{n} \beta_{n}$ and due to $\alpha_{n}=\left(f_{n}(x)-\beta_{n}\right) / x$ for $x>0$, there exists $\lim _{n} \alpha_{n}=\alpha_{0}$. Let $n, k$ be arbitrary integers, then $f_{n}(x) \leq f_{n+k}(x)=\alpha_{n+k} x+\beta_{n+k}$, 
so that $f_{n}(x) \leq \lim _{k} f_{n+k}(x)=\alpha_{0} x+\beta_{0}$. Hence, $f(x)=\alpha_{0} x+\beta_{0} \in E$. Moreover, $E$ is a lattice such that $f \wedge g \in E$ (with respect to the order by points) for every $f, g \in E$, but $\min \{f, g\}$ is not necessarily in $E$, so that $E$ is not a tribe.

We state that $s_{0}$ and $s_{1}$ are extremal states, but $s_{x} \notin \partial_{e} \mathcal{S}(E)$ for any $x \in(0,1)$ : Since $X$ and $\mathcal{S}(E)$ are affinely homeomorphic, $\partial_{e} \mathcal{S}(E)=\left\{s_{0}, s_{1}\right\}$ and $\mathcal{S}(E)=\left\{s_{x}\right.$ : $x \in[0,1]\}$.

(3) Let the effect-tribe be that from Example 4.3. Then every state $s_{x}$ for $x \in X$ is extremal, where $s_{x}(f)=f(x), f \in \mathcal{T}$. Indeed, let $x_{0}$ and $f, g \in G^{+}$be fixed. We verify criterion (5.2).

(i) Assume that $x_{0} \in X \backslash\{a, b\}$. Since $f, g$ are bounded, take $h \in G^{+}$such that $h(x)=0$ for each $x \neq x_{0}$ and $h\left(x_{0}\right)=\min \left\{f\left(x_{0}\right), g\left(x_{0}\right)\right\}$. By (5.2), $s_{x_{0}}$ is extremal.

(ii) Assume that $x_{0}=a$ and choose $h \in G^{+}$such that $h(a)=\min \{f(a), g(a)\}$, $h(b)=0, h(x)=(h(a)+h(b)) / 2=h(a) / 2$ for $x \in\left(A_{f} \cap A_{g}\right) \backslash\{a, b\}$ and $h(x)=0$ for otherwise. Again by (5.2), $s_{a}$ is extremal and the same true for $s_{b}$.

Nevertheless $\mu_{0}$ is extremal, the state $s_{\mu_{0}}$ is not extremal because $s_{\mu_{0}}=\left(s_{a}+\right.$ $\left.s_{b}\right) / 2$ but in view of Proposition 5.4, $s_{\mu_{0}}$ is a Jauch-Piron state.

(4) If we consider the tribe $\mathcal{T}(H)$, then every state $s_{x}\left(f_{A}\right)=(A x, x)$ is an extremal state when $\operatorname{dim} H \geq 3$. This follows from the Aarnes theorem and not from (5.2). because the state space of $\mathcal{E}(H)$ is not a simplex.

\section{States on Effect Algebras and Integrals}

In the present section, we show that any state on an effect algebra can be represented as an integral through a regular Borel probability measure. This will generalize the results from [Dvu4] where this was proved only for states on interval effect algebras.

We start with some definitions.

If $K$ is a compact Hausdorff topological space, let $\mathcal{B}(K)$ be the Borel $\sigma$-algebra of $K$ generated by all open subsets of $K$. Let $\mathcal{M}_{1}^{+}(K)$ denote the set of all probability measures, that is, all positive regular $\sigma$-additive Borel measures $\mu$ on $\mathcal{B}(K)$. We recall that a Borel measure $\mu$ is called regular if

$$
\inf \{\mu(O): Y \subseteq O, O \text { open }\}=\mu(Y)=\sup \{\mu(C): C \subseteq Y, C \text { closed }\}
$$

for any $Y \in \mathcal{B}(K)$.

The following result is motivated by research in $\mathrm{KuMu}$. Here we present another proof which is motivated by the theory of states on effect algebras.

Let $E \neq \emptyset$ and let $[0,1]^{E}$ be endowed with a product topology, i.e., with the weak topology, and let $\mathcal{S}$ be a nonempty closed convex subset of the Tikhonov cub $[0,1]^{E}$. Any map $s \in \mathcal{S}$ will called also a state. For any $a \in E$, let $\hat{a}(s):=s(a)$, $s \in S$. Then $\widehat{E}=\{\hat{a}: a \in E\} \subseteq \operatorname{Aff}(\mathcal{S})$.

Theorem 6.1. Let $s \in \mathcal{S}$ and let $\mathcal{S}$ be convex and closed in $[0,1]^{E}$. Then there is a regular Borel probability measure, $\mu_{s}$, on $\mathcal{B}(\mathcal{S})$ such that

$$
s(a)=\int_{\mathcal{S}} \hat{a}(x) \mathrm{d} \mu_{s}(x)
$$

for each $a \in E$. 
Proof. We define a congruence $\equiv$ on $E$ by $a \equiv b$ iff $s(a)=s(b)$ for any $a \in E$. Given $s \in \mathcal{S}$, denote by $\hat{s}$ the mapping from $E / \equiv$ into $[0,1]$ defined by $\hat{s}([a])=s(a)$, where $[a]$ denotes the congruence class corresponding to the element $a \in E$, and let $\widehat{\mathcal{S}}=\{\hat{s}: s \in \mathcal{S}\}$. Then the mapping $s \mapsto \hat{s}$ is an affine homeomorphism. Therefore, without loss of generality, we can assume that if $a \neq b$, then there is a state $s \in \mathcal{S}$ such that $s(a) \neq s(b)$.

Denote by $G$ the subgroup of $\operatorname{Aff}(\mathcal{S})$ generated by $\widehat{E}$, and let $E_{1}=\Gamma(G, 1)$ be the effect algebra generated by $\widehat{E}$.

We have that $\mathcal{S}$ and $\mathcal{S}(\operatorname{Aff}(\mathcal{S}), 1)$ are affinely homeomorphic under the mapping $\rho(s)(f)=f(s), f \in \operatorname{Aff}(\mathcal{S})$. Hence, the restriction of $\rho(s)$ onto $E_{1}$ is a state of the effect algebra $E_{1}$. Now let $t$ be a state on $E_{1}$. By [Goo, Cor 4.3] or by [Dvu4, Thm 6.9], this state can be extended to a state $\hat{t}$ on $\Gamma(\operatorname{Aff}(\mathcal{S}), 1)$ and to a state on $(\operatorname{Aff}(\mathcal{S}), 1)$. Hence, there is a mapping $s \in \mathcal{S}$ such that $\hat{t}=\rho(s)$, so that $t(g)=g(s)$ for any $g \in G$. Due to our assumption, we see that if $\rho\left(s_{1}\right)=\hat{t}=\rho\left(s_{2}\right)$, then $s_{1}=s_{2}$. Therefore, the mapping $t \in \mathcal{S}\left(E_{1}\right) \mapsto s$, where $s \in \mathcal{S}$ such that $\rho(s) \mid E_{1}=t$ is an affine homeomorphism; and we denote by $\theta: \mathcal{S} \rightarrow \mathcal{S}\left(E_{1}\right)$ the inverse affine homeomorphism.

So that, if $s \in \mathcal{S}$ and $a \in E$, then $s(a)=\hat{a}(s)=\rho(s)(\hat{a})$ and using [Dvu4, Thm 6.3], there is a regular Borel probability measure $\nu_{s}$ defined on $\mathcal{B}\left(\mathcal{S}\left(E_{1}\right)\right)$ such that

$$
s(a)=\rho(s)(\hat{a})=\int_{\mathcal{S}\left(E_{1}\right)} \widehat{\hat{a}}(y) \mathrm{d} \nu_{s}(y),
$$

where $\widehat{\hat{a}}$ is a mapping from $\mathcal{S}\left(E_{1}\right)$ into $[0,1]$ such that $\widehat{\hat{a}}(t):=t(\hat{a}), t \in \mathcal{S}\left(E_{1}\right)$. If we define a regular Borel measure $\mu_{s}=\nu_{s} \circ \theta$ defined on $\mathcal{B}(\mathcal{S})$, we have

$$
s(a)=\int_{\theta^{-1}\left(\mathcal{S}\left(E_{1}\right)\right)} \widehat{\hat{a}}(\theta(x)) \mathrm{d} \nu_{s}(\theta(x))=\int_{\mathcal{S}} \hat{a}(x) \mathrm{d} \mu_{s}(x) .
$$

Remark 6.2. If $\mathcal{S}$ is a Choquet simplex, then a regular Borel probability measure on $\mathcal{B}(\mathcal{S})$ in $(6.1)$ is a unique maximal regular Borel probability measure such that $\mu_{s} \sim \delta_{s}$ (see Dvu4, Thm 6.3]); if $\mathcal{S}$ is a Bauer simplex, then $\mu_{s}$ is a unique regular Borel probability measure, and $\mu_{s}\left(\partial_{e} \mathcal{S}\right)=1$.

In what follows, we show that (6.1) holds also for any nonempty compact set $\mathcal{S} \subseteq[0,1]$. We note that we will prove it using other argumentations than those used in Theorem 6.1

Theorem 6.3. Let $s \in \mathcal{S}$ and let $\mathcal{S}$ be closed in $[0,1]^{E}$. Then there is a regular Borel probability measure, $\mu_{s}$, on $\mathcal{B}(\mathcal{S})$ such that (6.1) holds for any $a \in E$ and $s \in \mathcal{S}$.

Proof. The mapping $\epsilon: \mathcal{S} \rightarrow K:=\mathcal{M}_{1}^{+}(\mathcal{S})$ defined by $\epsilon(s)=\delta_{s}$, where $\delta_{s}$ is the Dirac measure concentrated at the point $s$, is a homeomorphism from $\mathcal{S}$ onto $\partial_{e} \mathcal{M}_{1}^{+}(\mathcal{S})$.

Let $a \in E$, and let $a_{\epsilon}: \partial_{e} K \rightarrow[0,1]$ be defined by $a_{\epsilon}\left(\delta_{s}\right):=s(a)$. Then $a_{\epsilon} \in$ $\mathrm{C}\left(\partial_{e} K\right)$. Since $K$ is a simplex, [Goo, Cor 10.18], by the Tietze Theorem [Alf, Prop II.3.13], $a_{\epsilon}$ can be uniquely extended to an affine function $\hat{a}_{\epsilon} \in \Gamma(\operatorname{Aff}(K), 1)$ defined on $K$. If $\hat{a}: \mathcal{S} \rightarrow[0,1]$, where $\hat{a}(s):=s(a)$, then $\hat{a}_{e}(\epsilon(s))=\hat{a}(s)$ for each $s \in \mathcal{S}$. 
Since the effect algebra $\Gamma(\mathrm{C}(K), 1)$ is an MV-algebra, Dvu4, Thm 6.5] yields there exists a (unique) regular Borel probability measure $\nu_{s}$ on $\mathcal{B}(K)$ such that $\nu_{s}\left(\partial_{e} K\right)=1$ and

$$
s(a)=\hat{a}_{\epsilon}\left(\delta_{s}\right)=\int_{\partial_{e} K} \hat{a}_{\epsilon}(y) \mathrm{d} \nu_{s}(y) .
$$

If we set $\mu_{s}:=\nu_{s} \circ \epsilon$, then $\mu_{s}$ is a regular Borel probability measure on $\mathcal{B}(\mathcal{S})$. Therefore,

$$
s(a)=\int_{\epsilon^{-1}\left(\partial_{e} K\right)} \hat{a}_{\epsilon}(\epsilon(x)) \mathrm{d} \nu_{s}(\epsilon(x))=\int_{\mathcal{S}} \hat{a}(x) \mathrm{d} \mu_{s}(x)
$$

that finishes the proof.

Corollary 6.4. Let $s$ be a state on an effect algebra E. There is a regular Borel probability measure, $\mu_{s}$, on the Borel $\sigma$-algebra $\mathcal{B}(\mathcal{S}(E))$ such that

$$
s(a)=\int_{\mathcal{S}(E)} \hat{a}(x) \mathrm{d} \mu_{s}(x), \quad a \in E .
$$

If, in addition, $\mathcal{S}(E)$ is a Choquet simplex, then there is a unique regular Borel probability measure, $\mu_{s}$, on $\mathcal{B}(\mathcal{S}(E))$ such that $\mu_{s}\left(\partial_{e} \mathcal{S}(E)\right)=1$ and

$$
s(a)=\int_{\partial_{e} \mathcal{S}(E)} \hat{a}(x) \mathrm{d} \mu_{s}(x), \quad a \in E .
$$

Proof. It follows from Theorem 6.1 and Remark 6.2.

\section{REFERENCES}

[Alf] E.M. Alfsen, "Compact Convex Sets and Boundary Integrals", Springer-Verlag, Berlin, 1971.

[AlSc] E.M. Alfsen, F.W. Schultz, "State Spaces of Operator Algebras", Birkhäuser, 2001.

[BeFo] M.K. Bennett, D.J. Foulis, Phi-symmetric effect algebras, Found. Phys. 25 (1995), 16991722 .

[BiNe] G. Birkhoff, J. von Neumann, The logic of quantum mechanics, Ann. Math. 37 (1936), 823-834.

[BrRo] O. Bratteli, D.W. Robinson, "Operator Algebras and Quantum Statistical Mechanics," Springer-Verlag, New York, Heidelberg, Berlin, 1979.

[BCD] D. Buhagiar, E. Chetcuti, A. Dvurečenskij, Loomis-Sikorski representation of monotone $\sigma$-complete effect algebras, Fuzzy Sets and Systems 157 (2006), 683-690.

[BCD1] D. Buhagiar, E. Chetcuti, A. Dvurečenskij, Loomis-Sikorski theorem and Stone duality for effect algebras with internal state, http://arxiv.org/abs/1006.0503

[BuKl] D. Butnariu, E.P. Klement, "Triangular Norm based Measures and Games with Fuzzy Coalitions," Kluwer Acad. Publ., Dordrecht, 1993.

[Cha] C.C. Chang, Algebraic analysis of many valued logics, Trans. Amer. Math. Soc. 88 (1958), 467-490.

[Dvu] A. Dvurečenskij, "Gleason's Theorem and Its Applications", Kluwer Academic Publisher, Dordrecht/Boston/London, 1993, 325+xv pp.

[Dvu1] A. Dvurečenskij, Central elements and Cantor-Bernstein's theorem for pseudo-effect alegbras, J. Austral. Math. Soc. 74 (2003), 121-143.

[Dvu2] A. Dvurečenskij, States on pseudo-effect algebras with general comparability, Kybernetika 40 (2004), 397-420.

[Dvu3] A. Dvurečenskij, Loomis-Sikorski theorem for monotone $\sigma$-complete effect algebras, J. Austral. Math. Soc. 79 (2005), 305-318.

[Dvu4] A. Dvurečenskij, Every state on interval effect algebra is integral, arXiv:1005.0171

[DvPu] A. Dvurečenskij, S. Pulmannová, "New Trends in Quantum Structures", Kluwer Acad. Publ., Dordrecht, Ister Science, Bratislava, 2000. 
[FoBe] D.J. Foulis, M.K. Bennett, Effect algebras and unsharp quantum logics, Found. Phys. 24 (1994), 1325-1346.

[Gle] A.M. Gleason, Measures on the closed subspaces of a Hilbert space, J. Math. Mech. 6 (1957), 885-893.

[Goo] K.R. Goodearl, "Partially Ordered Abelian Groups with Interpolation", Math. Surveys and Monographs No. 20, Amer. Math. Soc., Providence, Rhode Island, 1986.

[Hal] P.R. Halmos, "Measure Theory", Springer-Verlag, New York, Heidelberg, Berlin, 1988.

$[\mathrm{KuMu}]$ J. Kühr, D. Mundici, De Finetti theorem and Borel states in [0,1]-valued algebraic logic. Inter. J. Approx. Reasoning 46 (2007), 605-616.

[Kro] T. Kroupa, Every state on semisimple MV-algebra is integral. Fuzzy Sets and Systems 157 (2006), 2771-2782.

[Mun] D. Mundici, Interpretation of $A F C^{*}$-algebras in Eukasiewicz sentential calculus, J. Funct. Anal. 65 (1986), 15-63.

[Pan] G. Panti, Invariant measures in free MV-algebras, Comm. Algebra 36 (2008), 28492861.

[Rav] K. Ravindran, On a structure theory of effect algebras, PhD thesis, Kansas State Univ., Manhattan, Kansas, 1996.

[Rie] Z. Riečanová, A generalization of blocks for lattice effect algebras, Inter. J. Theoret. Phys. 39 (2000), 231-237. 